\title{
PHYSIOLOGICAL IMPLICATIONS OF PSYCHOPHYSICAL DATA*
}

\author{
Wilson P. Tanner, Jr. \\ The University of Michigan, Ann Arbor, Mich.
}

\section{Introduction}

In this paper I am presenting an approach to the study of psychophysics that includes the use of mathematical models. While the individual experiments can each be interpreted as a contribution to an area in psychophysics, the program can be interpreted as an experiment testing the feasibility of a profitable use of a particular scientific philosophy in the study of an area of psychology.

This philosophy involves the use of mathematical models in a manner similar to that of the physicist. It employs, first, the use of simple models describing the relations of only a few observable variables. The measures describing the relations among these variables may be measures of variables that exist only as force and gravity in physics and that can be measured only by observing their consequences. As measurement techniques become more nearly precise or, as the conditions of the experiment are extended to include a wider range, it will likely become apparent that the model had been adequate only because it was tested with coarse measurement over a restricted range of conditions. At this point it will be necessary to expand or modify the model to include the new data.

The expanded or modified model should still include the original model as applied to the limited conditions. FIGURE 1 is intended to illustrate this point. The large space is one that includes all possible data that might be relevant to a particular area of science. Many of the points in this space will never appear as data for they are contradictory to the "truth," whatever that may be. The shaded area in the center represents the data at hand. A model describing these data could be expanded in any of a number of directions. The large areas $A, B$, and $C$ represent models, each of which includes the original model. If the next datum collected turns out to be at point 1 , model $C$ can be eliminated from consideration. If it is collected at point $2, A$ and $C$ can be eliminated. Either point, however, requires an expansion of the original model.

We have tried to work with a general model that includes all of the points in the space. It is the general model of testing statistical hypotheses. The model can be expanded to specify the data that must appear if a sensory threshold exists, if Luce's ${ }^{3}$ axiom 1 is valid, or if the observer is intelligent. The general theory can be made specific in a number of ways; it is an experimental question that determines precisely how it should be made specific.

I shall trace one phase of the history of this program, showing how we introduced the concept of memory to modify the model to incorporate a wider

* The work described in this paper was supported in part by the United States Air Force under Contract No. AF49(638)-369, monitored by the Office of Scientific Research of the Air Research and Development Command, Wright Air Force Base, Dayton, Ohio. 
range of conditions. At times I shall try to point out some of the physiological implications. However, one must not forget that I am talking about concepts that are unobservable, and the physiologist may find himself faced with similar problems to those of the physicist chasing electrons: he may have to devise physiological cloud chambers so that his electrons will create an observable phenomenon. The nerve impulse may not be sufficient.

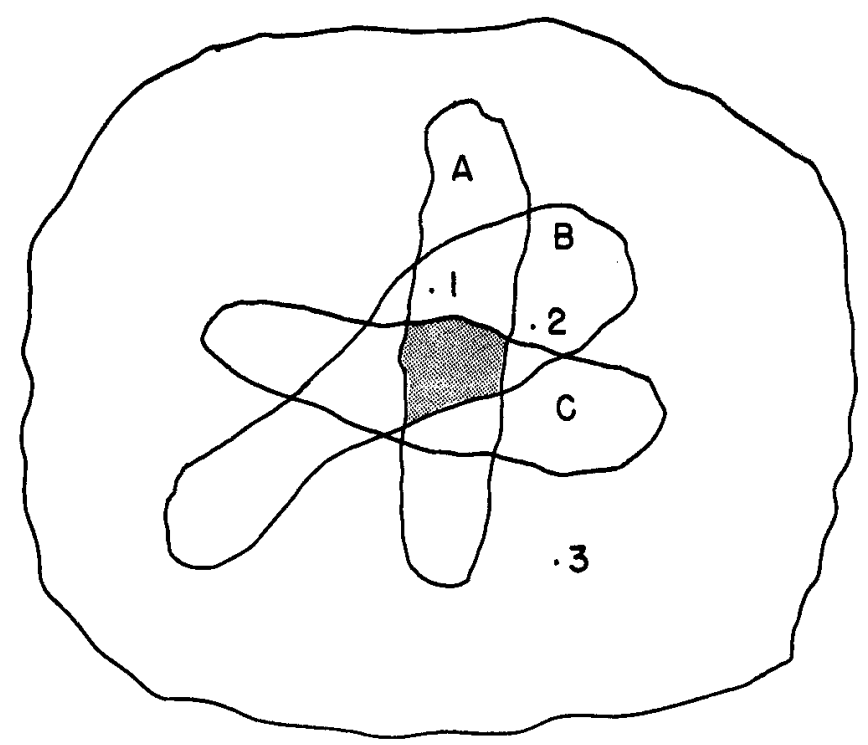

Figure 1. Theory space.

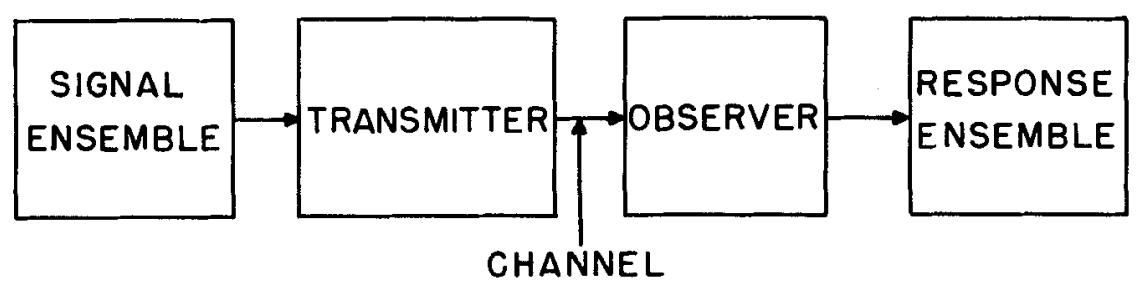

FIGURE 2. Block diagram of a typical psychophysical experiment.

\section{What is a Psychophysical Experiment?}

Psychophysics is the study of the relations of psychological quantities to physical quantities. A block diagram of a typical experiment is shown in FIGURE 2. The experimenter designates an observation interval in time, during which one of the signals from the ensemble is transmitted over the channel. At the end of the observation interval, the observer is asked to choose one of a set of responses. The joint event of the signal transmitted and the signal received (as indicated by the response) is recorded. 


\section{What are the Measures?}

In the simplest type of psychophysical experiment, in which a particular signal or nothing is transmitted during the observation interval, the data may be summarized as illustrated in FIGURE 3 . In this figure, the rows represent the transmitted events as modified by the channel. If the signal is transmitted, the observer's input is the signal as randomly perturbed by the channel. Here we are calling this input "signal plus noise." If nothing is transmitted, the observer's input is labeled "noise."

The columns are the observer's responses. "Yes" means that he considers the input to have arisen from signal plus noise, while "No" means that he considers the input to have arisen from noise alone. The data can be summarized as percentages that, in analysis, are assumed to be estimates of probabilities. Two conditional probabilities are estimated: the probability that if the signal is transmitted the observer's input will lead to a "yes" response,

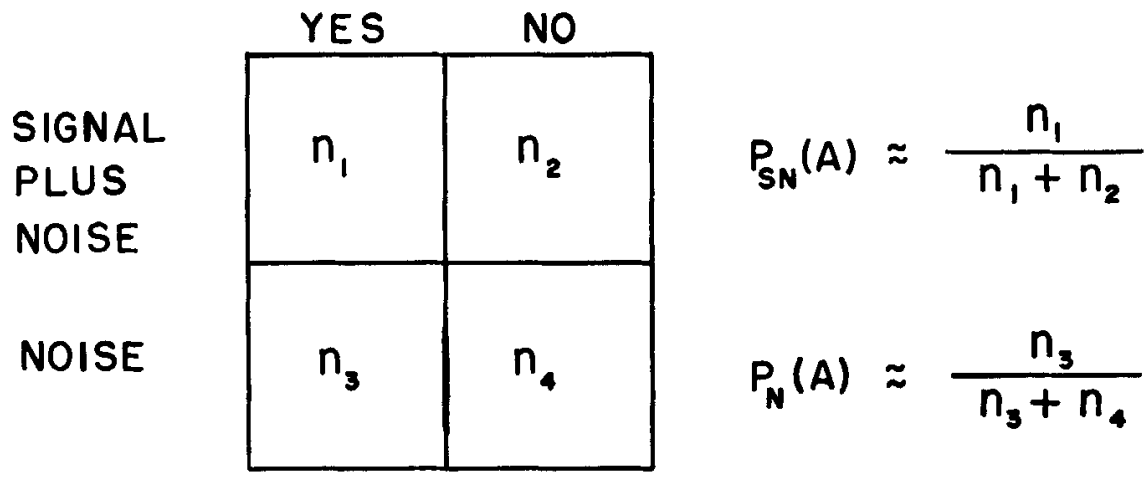

Figure 3. Data summary of a typical psychophysical experiment.

$P_{\mathrm{s} N}(A)$; and the probability that if nothing is presented, the observer's input will lead to a "yes" response, $P_{N}(A) . P_{s_{N}}(A)$ is the detection probability and $P_{N}(A)$ is the false-alarm probability.

In FIGURE 3 ,

$$
P_{S N}(A) \approx \frac{n_{1}}{n_{1}+n_{2}}
$$

and

$$
P_{N}(A) \approx \frac{n_{3}}{n_{3}+n_{4}}
$$

where the $n$ 's represent a counting of the various joint events occurring during the experiment.

The fact that we summarize the data as estimates of probabilities implies that the tasks the observer is asked to perform in psychophysical experiments are tasks in which there is a probability of error; consequently they satisfy Middleton's criterion (personal communication) as tasks that can be handled 
within the framework of decision theory. According to this criterion, whenever one is confronted with a probability of error he is confronted with a decision theory problem.

\section{Decision Theory Model}

Decision theory serves as a foundation for the theory of signal detectability; a theory that deals with exactly the problem with which the observer is confronted in a psychophysical experiment. An examination of the general theory of signal detectability may help us understand the observer's problem.

The problem handled in this theory is that in which an observer is asked to state which of two conditions led to a particular input, $x$, during a fixed observation interval, 0 to $T$. Any input $x$ is considered to be a function of time, $x(l)$, since time is the dimension of the observation interval. With each $x(l)$ two numbers are associated: the likelihood of that particular $x(t)$, if the signal was transmitted, $f_{S N}[x(t)]$; and the likelihood of that particular $x(t)$, if nothing was transmitted, $f_{N}[x(t)]$. The decision theory problem is to select a criterion space, $A$, which includes all of those $x(l)$ s that should lead to the response "yes" and excludes all of those $x(t)$ s that should lead to the response "no."

This can be stated as the problem of choosing the criterion space $A$ such that

$$
P_{S N}(A)-W P_{N}(A)=a \max
$$

where $W$ is a number or weighting function.

The following proof, derived by Fox, ${ }^{1}$ demonstrates that the likelihood ratio describes the information contained in the input, $x(t)$, relevant to the choice the observer must make. Equation 1 can be written as

$$
\int_{A} f_{S N}[x(t)] d x(t)-W \int_{A} f_{N}[x(t)] d x(t)=a \max .
$$

Since both variables are over the same space, this becomes

$$
\int_{A} f_{S N}[x(t)] d-W f_{N}[x(t)] d x(t)=a \max .
$$

In this form it becomes obvious that the integral is a maximum if all positive values are included and no negative values are included. Thus the space, $A$, should include all of those values of $x(t)$, and only those values, which satisfy the following inequality:

$$
f_{S N}[x(t)]-W f_{N}[x(t)] \geq 0
$$

or

$$
l[x(t)]=\frac{f_{\mathrm{SN}}[x(t)]}{f_{N}[x(t)]} \geq W
$$

where $l[x(t)]$ is defined as the likelihood ratio. 


\section{What is an Ideal Observer?}

Given Fox's proof, we are now in a position to define an ideal observer, illustrated in its general form in FIGURE 4 . It is an observer who computes the likelihood ratio associated with the input, $x(l)$, and then matches this value to a point in the criterion space. If the point is contained in $A$, then it says "yes," the signal was transmitted. If it is not in $A$, then it is in the complement of $A, C A$; and the observer says "no," the signal was not transmitted. The computation of $l[x(t)]$ and the matching are performed without error.

If we were to attempt to designate in detail the construction of an ideal observer, it would be necessary to know the signals that might be transmitted,

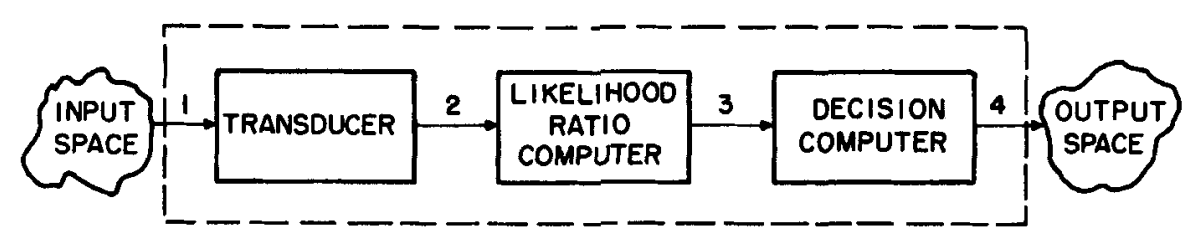

Figure 4. Ideal observer.

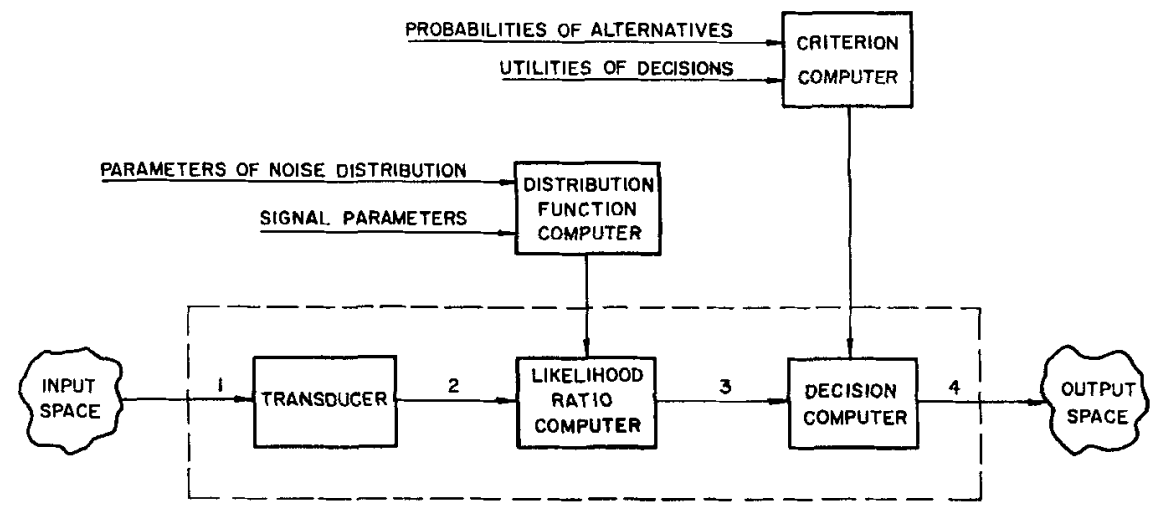

Figure 5. The ideal observer with tie-ins to the system.

the properties of the perturbing noise, the way in which the signal and noise are combined, and the function $W$, to be maximized. All of these parameters are required to specify the specific components (such as filters and integrators), and the particular criterion spaces, $A$ and $C A$. Any change in the values of parameters can lead to a change in the detailed designation of the ideal observer. Thus if we want to consider the ideal observer as applied to specific situations, then there is an ideal observer for each specific case. They are all alike in that they compute likelihood ratios and say "yes" if the likelihood is greater than $W$. Whether any specific $x(t)$ leads to a "yes" or a "no" response depends on the signal and noise conditions from which it might have arisen and on the particular function, $W$, used in the maximization.

The whole operation is illustrated in FIGURE 5. The part of the block diagram included in the dotted lines can be considered as an ideal sensory system. 
Those parts outside the dotted lines are the connecting links between the sensory system and the larger system that the sensory system serves. The connecting links mold the sensory system into an ideal form for the particular task at hand. It sets the parameters of the components and specifies the criterion spaces in a way that leads to optimum performance.

\section{The Human Observer and the Ideal Observer}

We probably all agree that, in the strict sense of the word, the human observer cannot be considered as an ideal observer. Few of us would expect perfect computations of likelihood ratios and perfect matching into the criterion spaces.

However, we might feel that the human observer's sensory system is, in a broad sense, similar to that of an ideal observer in some respects. It is, after all, a subsystem of an "intelligent" system and it seems not unlikely that the intelligent system would have some control over the form the subsystem assumes in facing any particular task. It may be molded in a way such that it exhibits a phenomenon we frequently refer to as "attention." Its performance may vary in a way that is consistent with different methods for computing $W$. While it may not achieve an ideal performance, it may in some ways tend toward an ideal performance.

If this is the case, then the experimenter must interpret his results in terms of the particular experimental conditions that have the potential of leading to a modification of the system. If these modifications are possible, then the physiologist is faced with a severe task in attempting to interpret data collected in experiments that eliminate the effects of the modifying agents. This line of reasoning clearly indicates physiological studies employing "consciously" behaving animals with implanted electrodes: the type of study I have heard Walter Rosenblith advocate many times.

FIGURE 6 shows a block diagram of an observer based on the ideal observer, but it also shows some of the places in which one might expect imperfections to exist. This diagram has as its basic structure that of the ideal observer, and the same links connecting the observer to the larger system. It has had added to it a number of memory banks that are immediately suggestive of imperfections. Let us examine the various properties an ideal memory must have in a simple detection experiment where the signal is a segment of a sine wave. This signal is specified exactly; thus when it occurs in the observation interval, 0 to $T$, it will occur at a precisely specified position within the interval. Its frequency, phase, and amplitude will also be specified precisely. This means that the function of time representing the signal can be positioned exactly in the observation interval. The value of this function can be precisely stated for any moment of time in the observation interval.

To be able to do this, an observer must have stored in his memory a precise representation of the function of time. He needs a clock mechanism that permits him to synchronize exactly with the observation interval, 0 to $T$, and to locate exactly in the interval the point at which the function of time should start, and precisely its duration. His memory must specify exactly the frequency, phase, amplitude, starting time, and duration of the segment of the sine wave. Any error in the memory will lead to a decrement in performance. 
It is possible to make some inferences from psychophysical experiments regarding the role that each of these aspects of memory plays in the human observer's performance. It must be remembered, however, that these inferences are based on a model designed to describe the relation between the observables in a psychophysical experiment: the transmitted signals and the responses. The models can be looked at as mathematical equations fitting the data. As long as the data are finite, there are an infinite number of models that will fit equally well. Data collected in the future may eliminate this model, but will never confirm it. It uses words such as "memory" for the parameters because these words have intuitive appeal. The physiologist

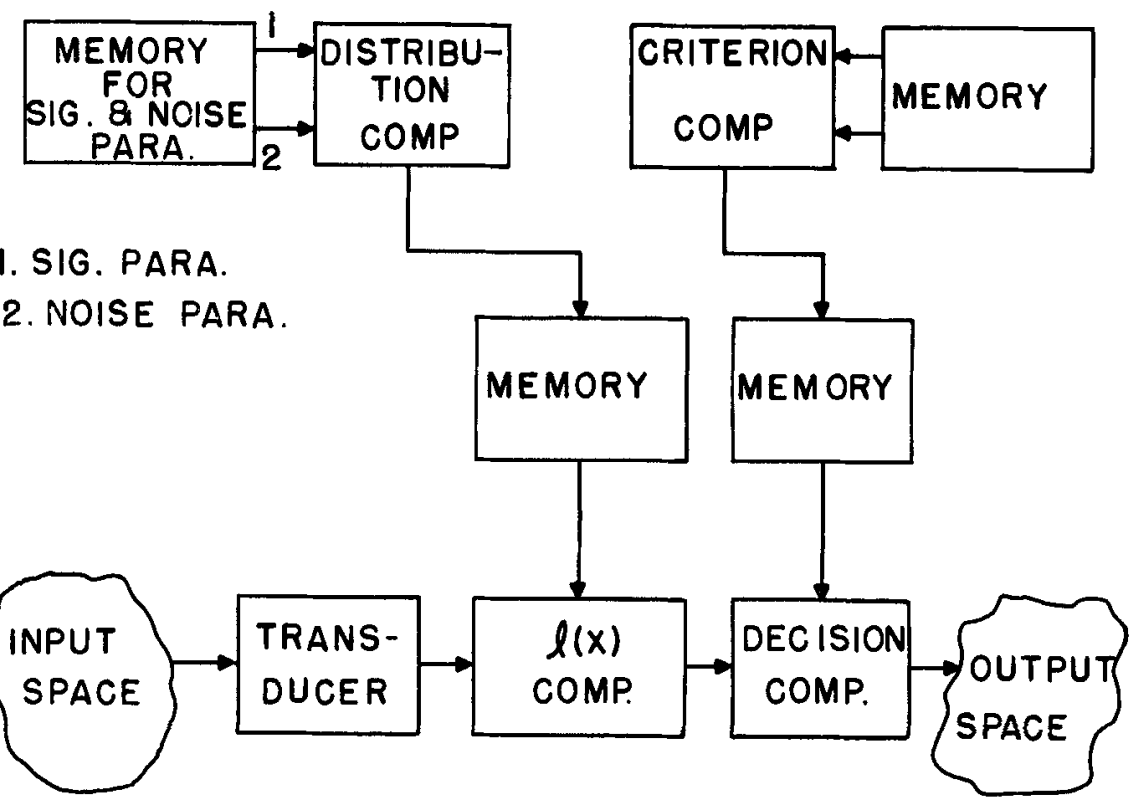

FIgURE 6. The ideal observer modified to include memory.

trying to correlate his observations with the parameters of the model must remember that the concept of memory arises from the model, not the data.

\section{A Psychophysical Investigation of "Memory"}

One of the concepts involved in using the theory of signal detectability as a tool for interpreting psychophysical data is expressed in the following "theorem":

If in two channels, one employing an ideal transmitter and an imperfect observer; and the other a transmitter with statistical uncertainty and an ideal receiver, both transmitters having equal energies available, the performance as indicated by measures of the false-alarm rate and the detection rate are found to be identical, then the imperfect receiver can be said to introduce the same degree of uncertainty in its channel as the transmitter in the other channel. 
The uncertainty attributed to the receiver, it was felt, would reflect the observer's memory. Consequently a series of models with specified transmitter uncertainties were studied, leading to calculations of the performance measure $d^{\prime}$ as a function of $2 E / N_{0}$. Figure 7 shows these plotted on log-log paper, and a human observer's data superimposed. The notion was that if the hu-

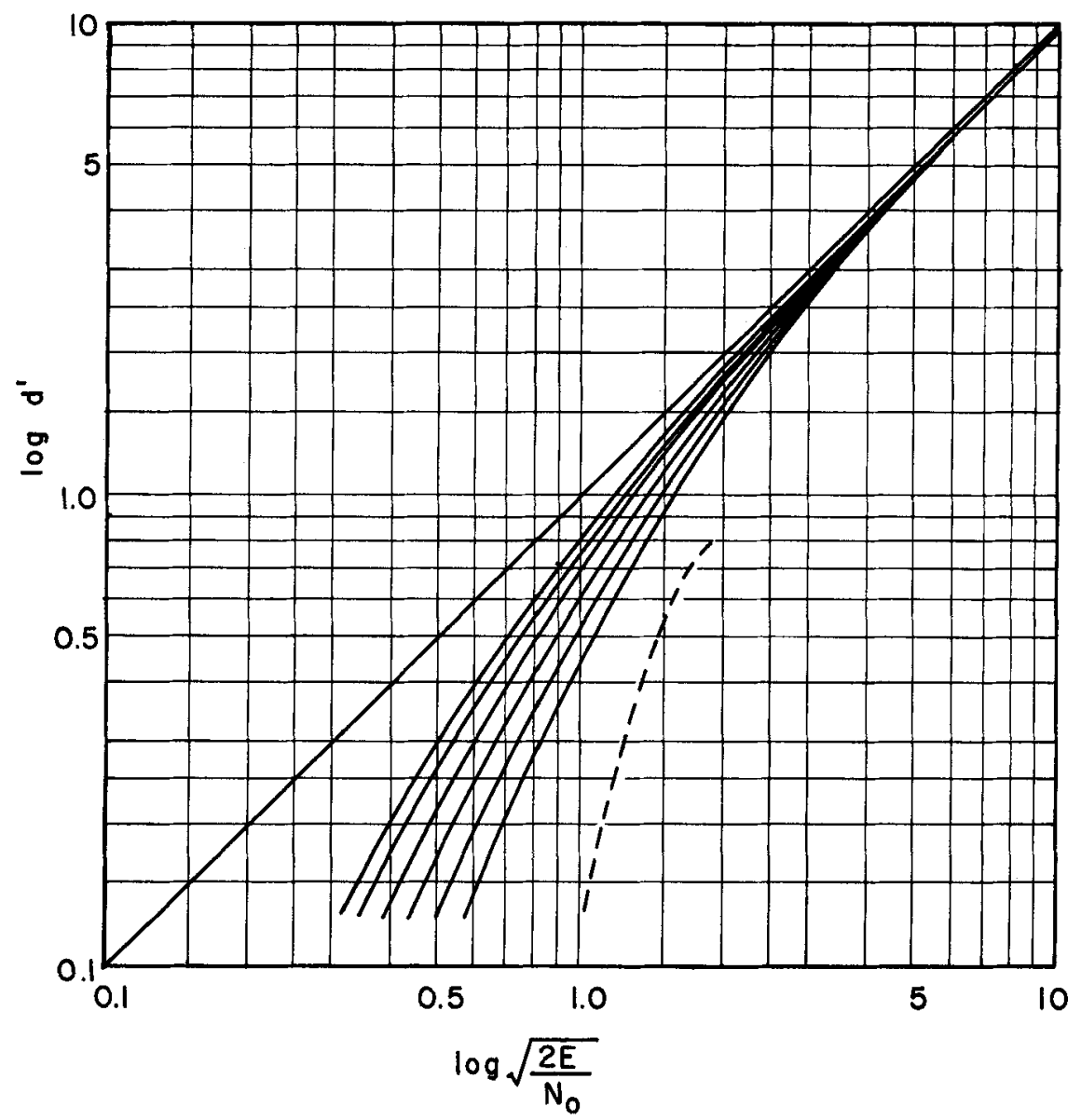

Figure 7. Performance curves for signals specified statistically.

man observer's data matched one of the curves, the degree of uncertainty introduced by him could be specified, and his memory characterized by a number.

The concept is far too simple. The human observer's data did not fall on any one curve, but rather cut across curves. This suggested that a different model would be required for every signal level, at least in terms of the number categorizing the memory.

Careful examination of the experimental procedures under which the data were collected suggests that this is not unreasonable. Each signal level was 
studied in a sequence of 100 observations coming about five seconds apart. Before the sequence, the observer was permitted to listen to several samples of the signal without noise, and these noiseless samples were assumed to establish his memory.

If the signals are weak, then, as the sequence proceeds, the observer will receive few samples that are clear enough to reinforce his memory. If they are strong, he is more likely to receive some fairly good samples, and his memory on the average should be better throughout the sequence than it would be for the weak signals.

Elizabeth Shipley (personal communication) has recently performed an analysis of some data in which she considers the probability of a correct choice, given that the previous choice was correct and given that the previous choice was incorrect. She finds that a dependency exists when the signals are segments of sine waves, and does not exist when the signals are samples of noise. The dependency is in the right direction to support the hypothesis that a clear sample tends to reinforce the memory.

When Julian Bigelow saw some of the early results, he suggested a way of attacking the problem experimentally. He suggested that a sine wave of the same phase and frequency as the signal be added as a component of the noise, replacing the need for the observer's memory of frequency and phase. The signal would be superimposed on the sine wave during the observation interval. This procedure altered the slope of the psychophysical function so that it agreed with one of the slopes of the computed curves of fIGURE 7 . However, it is positioned wrong.

There was still a need for the observer to have a memory for starting time and duration. My colleagues and I were able to extend Bigelow's suggestion in a way that could remove the need for this memory. This we have referred to as a "pulsed carrier" experiment. In each observation interval we introduced a segment of sine wave whose amplitude was large. This segment matched the signal in frequency, phase, starting time, and duration. It was the observer's task to state whether the signal appeared on top of this pulsedcarrier or pedestal, as J. C. R. Licklider calls it. In this case, the psychometric function turns out to be linear, or as if there were some added noise.

This leads to an interpretation of the memories required as a function of the experimental design, as illustrated in TABLE 1. There are three types of experiments: detection, continuous wave, and pulsed-carrier; each with two subdivisions, "yes-no" and $2 \mathrm{AFC}$. The detection experiment requires a memory for frequency, phase, starting time, and duration. The CW experiment requires only starting time and duration memories, while the pulsed-carrier experiment requires none of these. The difference between a "yes-no" experiment and a 2AFC experiment is the need for amplitude memory in the "yesno" experiment.

A summary of the results of the various experiments follows:

(1) In the detection experiment, the slope of the psychometric function is too great to be accounted for in terms of a fixed uncertainty. There is no difference between a "yes-no" and a 2AFC experiment, probably because the other memories are so poor that they mask the effect of amplitude memory. 
(2) In the CW experiment, the slope of the psychometric function is such that it can be accounted for in terms of a fixed uncertainty. The function is misplaced, prohibiting an estimate of the degree of uncertainty. There is little, if any, difference between "yes-no" and $2 \mathrm{AFC}$ experiments.

(3) In the PC experiments, $d^{\prime}$ is a linear function $\left(2 E / N_{0}\right)^{1 / 2}$, a condition that can be accounted for by additive noise. Performance in 2 AFC experiments is noticeably superior to that in "yes-no" experiments.

The analysis of the memory ccnditions shown in TABLE 1 is not precisely true for an imperfect observer, who for example must have some amplitude memory in the $2 \mathrm{AFC}, \mathrm{PC}$ experiment and must be able to remember the amplitude of the waveform in the first interval so that he can compare it to the amplitude of that in the second interval. In fact, the experiment can be looked at as a "yes-no" experiment in which the measure established in the first interval serves as a cut-off for the second. If the intervals are widely

TABI.E 1

Memories Required for Different Psychophysical Experiments

\begin{tabular}{|c|c|c|c|c|c|c|}
\hline & & \multicolumn{5}{|c|}{ Required memories } \\
\hline \multicolumn{2}{|c|}{ Experiments } & & 1 & & Starting & \\
\hline Detection & $\begin{array}{l}\text { "Yes-No" } \\
2 \text { AFC }\end{array}$ & + & $\begin{array}{l}+ \\
+\end{array}$ & $\begin{array}{l}+ \\
+\end{array}$ & $\begin{array}{l}+ \\
+\end{array}$ & + \\
\hline Continuous wave & $\begin{array}{l}\text { "Yes-No" } \\
2 \mathrm{AFC}\end{array}$ & + & & & $\begin{array}{l}+ \\
+\end{array}$ & + \\
\hline Pulsed carrier & $\begin{array}{c}\text { "Yes-No" } \\
2 \text { AFC }\end{array}$ & + & & & & \\
\hline
\end{tabular}

separated, this cut-off would not be precisely remembered. It might be as if noise were added to this cut-off, the amount of noise depending directly on the time between the two pulses.

Cut-off variance can be treated as additive noise, provided that all of the prior stages are linear, as illustrated in FIGURE 8. There are three distributions shown: in each the abscissa is the value of the decision variable, and the ordinate is the probability density of that variable. The distribution to the left is for the second signal, given that it is the smaller of the two. The distribution to the right is for the second signal, given that it is the larger. The middle distribution is the critical value of the decision variable, $x_{c}$.

It is possible to transform to a new decision axis, $x-x_{c}$, where on this new axis there is a fixed cut-off at 0 . There are now only two distributions, $x-x_{c}$ given the smaller signal, and $x-x_{c}$ given the larger signal. Each of these has added the variance of $x_{c}$ to its original variance.

Using the form of the psychometric function for the pulsed-carrier to demonstrate that the stages prior to the cut-off are linear, it should now be possible to study the variance of the cut-off as a function of the time, $T$, between the observation intervals. One complication still exists, however: there must 
be a transducer type of operation at the input and, if $T$ is too small, independent observations cannot be expected. Thus the performance should drop both for very small values of $T$ and for very large values of $T$. In between there should be a peak. In fact, the following equation should specify $d^{\prime}$ :

$$
\left(d^{\prime}\right)^{2}=\eta_{0}\left(1-e^{-e T}\right) \frac{2 E_{\Delta}}{N_{0}+k T}
$$

where $e$ is something like the time constant of the transducer, $k$ is the noise energy per unit time added to the cut-off value, and $\eta_{0}$ represents the efficiency if added noise of the nervous system alone accounts for the fact that the efficiency is less than unity. A typical set of results is shown in FIGURE 9.
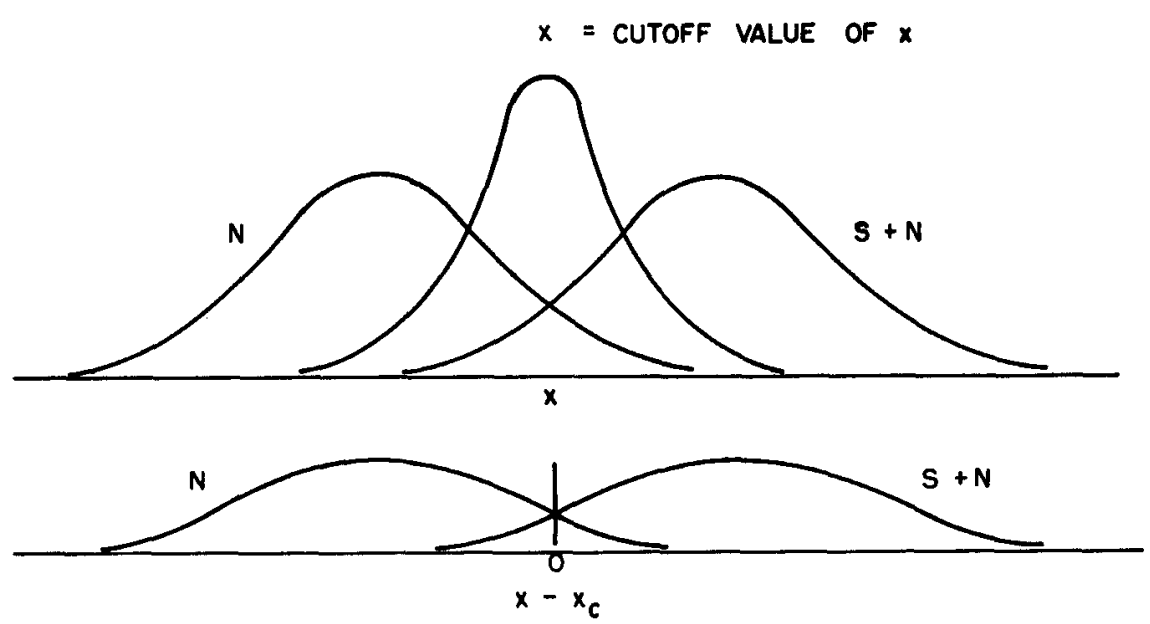

FigURE 8. Illustration of the cut-off variance as added noise.

The CW experiment, using the same observer, may be re-examined now. Suppose it is assumed that the value of $2 E / N_{0}$ used in the experiment has been reduced to the value of $\left(d^{\prime}\right)^{2}$, as calculated by the equation with the parameters determined by the pulsed-carrier experiment. One may now plot the psychometric functions as $\left(d^{\prime}\right)^{2}$ versus the corrected value of $2 E / N_{0}$ (FIGURE 10). The misplacement of the curve is now corrected so that it is possible to estimate the degree of statistical uncertainty introduced by faulty memories of starting time and duration. The values of this parameter for the four observers range from about 5 to approximately 12 .

All of the experiments thus far discussed have involved acoustic signals. My colleagues and I have also made visual studies, and R. Fitzhugh ${ }^{2}$ at the Johns Hopkins University, Baltimore, Md., has reported some physiological data related to these studies. At first appearance there seems to be a discrepancy between Fitzhugh's data and ours. However, the discrepancy disappears when it is considered in terms of the memory framework.

Fitzhugh studied the frequency of nerve impulses on the dark-adapted cat's eye, counted over an arbitrary time interval after a flash of light had been 


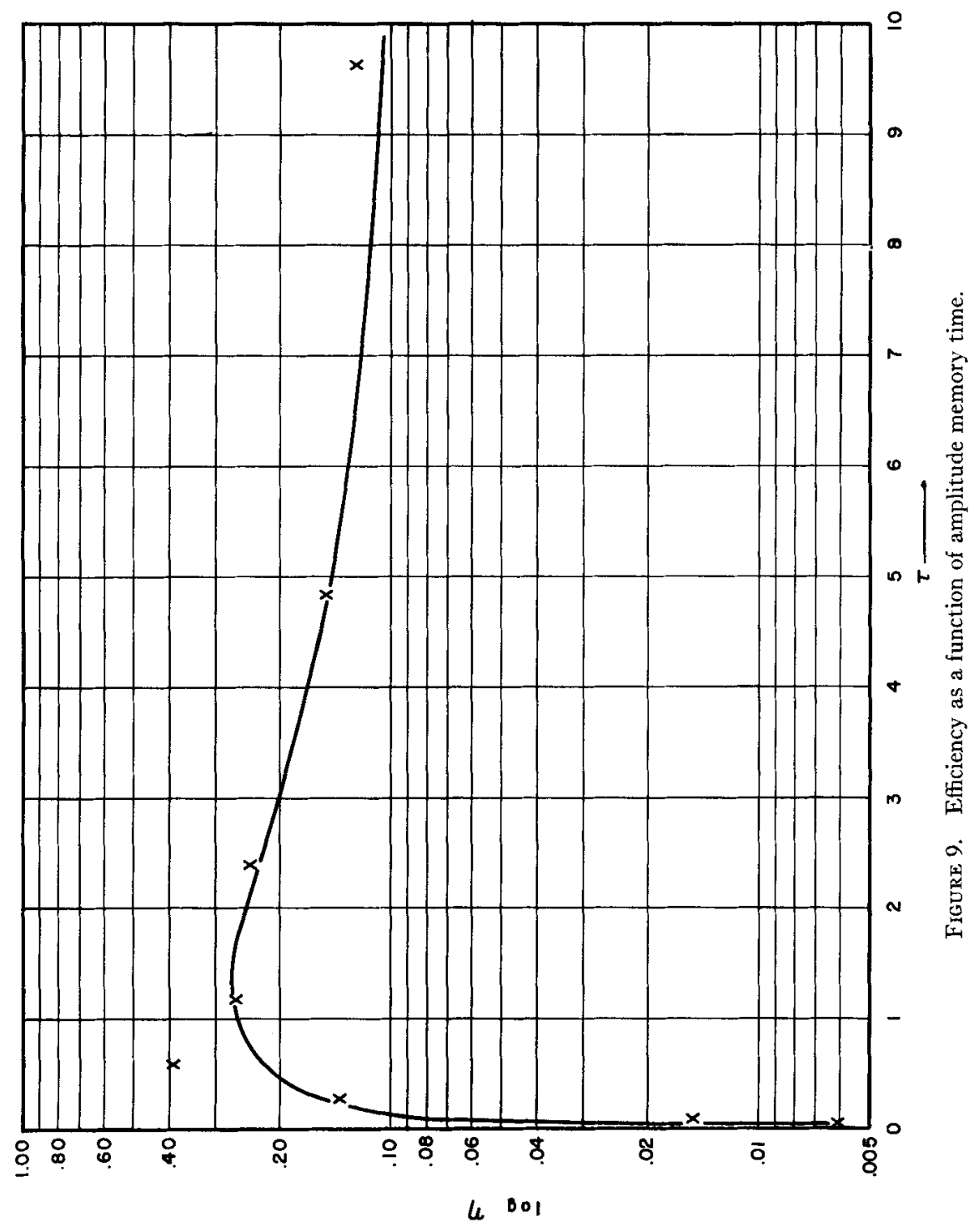




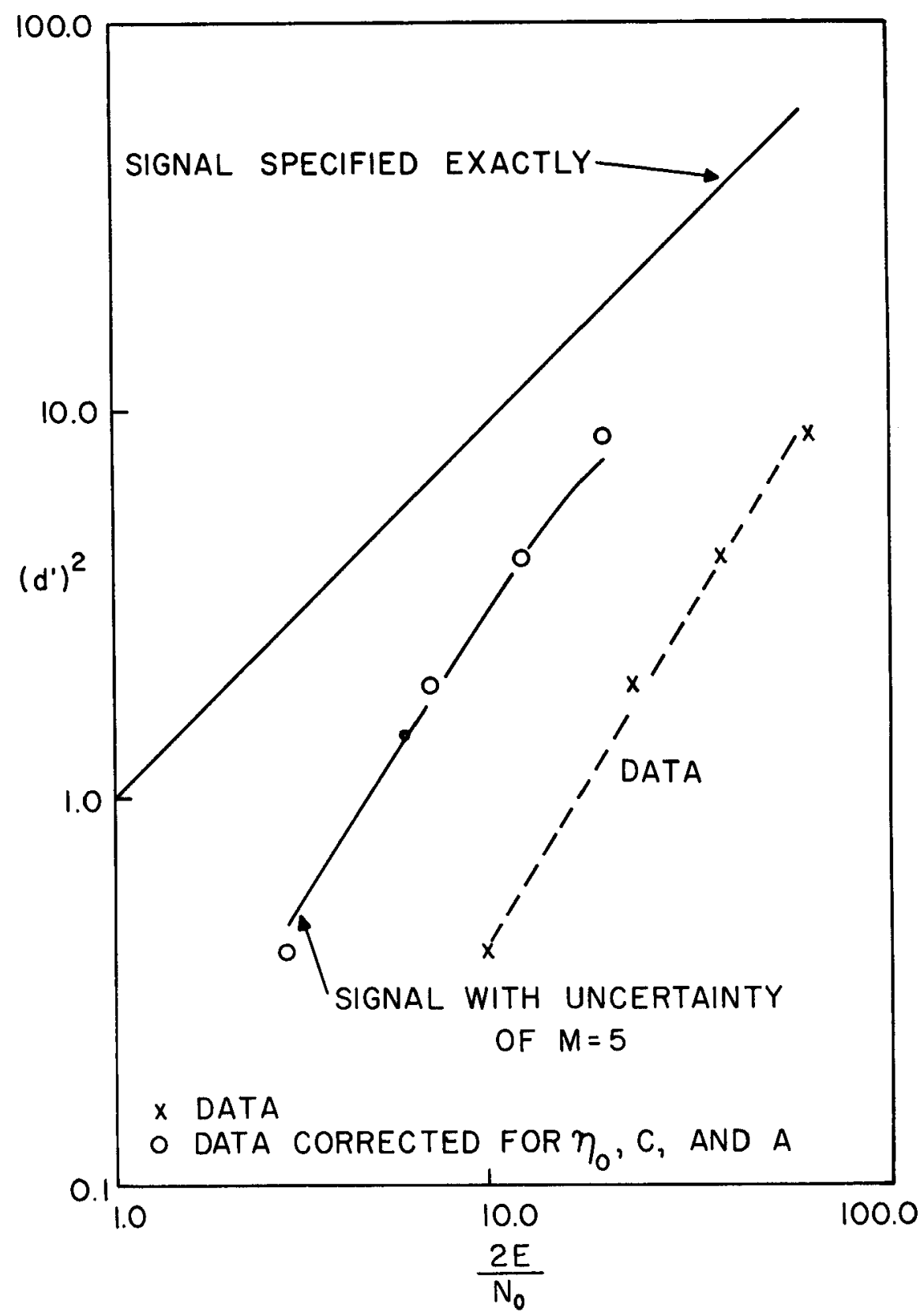

FIGURE 10 Data corrected to permit an estimate of starting time and duration uncertainty. 
introduced. From his studies he was able to construct statistical distributions associated with each of a number of intensities (including 0 or noise alone). He could then assign values of $d^{\prime}$ representing the separation between any one of these distributions and that for noise alone. According to Fitzhugh, $d^{\prime}$ was approximately linearly related to $\Delta \mathrm{I}$, while in our psychophysical experiments $d^{\prime}$ varied as $\Delta \mathrm{I}^{p}$, where $p$ was 2 or greater. An examination of our procedure indicates that there might be uncertainty both as to the time and location of the signal presentation. The slope of our curves agreed with the slope of the curves for $M=100$. The uncertainty may be introduced at a central rather than retinal level, suggesting that one should not try to account for nonlinearities at the level of the end-organ, to the exclusion of central hypotheses.

One more point on the relation between Fitzhugh's results and ours. We analyzed our results on the assumption that normal distributions existed. Fitzhugh's distributions started at zero, and appeared quite skewed. This is not a discrepancy, for, from the standpoint of psychophysical analysis, any distribution that can be made normal by means of a monotonic transformation of the decision variable can be analyzed in a fashion consistent with the normal assumption.

To demonstrate this point, let us consider Fox's proof, which shows that likelihood ratio is the proper decision variable. Likelihood ratio varies from 0 to infinity and, for noise alone, at least, is highly skewed. However, if one considers the decision rule "say yes" if $\log _{e} l(x) \geq \log _{e} W$, every decision is the same as if it were made according to the decision rule "say yes" if $l(x) \geq$ $W$. $\log _{e} l(x)$ varies from $-\infty$ to $+\infty$, and under certain conditions is normal. Since all the decisions are the same, and since one can only observe the decisions, a normal analysis can be applied whether the decision variable is $\log l(x)$ or $l(x)$. The results will be the same in both cases.

\section{References}

1. Fox, W. C. 1953. Signal detectability: A unified description of statistical methods employing fixed and sequential observation processes. TR-19. Electronic Defense Group, Univ. Michigan. Ann Arbor, Mich.

2. Fitzhugh, R. 1957. The statistical detection of threshold signals in the retina. J. Gen. Physiol, 40: 925-948.

3. LuCE, R. D. 1959. Individual Choice Behavior. : 6. John Wiley. New York, N. Y. 